\section{DEUTSCHE FACHGESELLSCHAFT

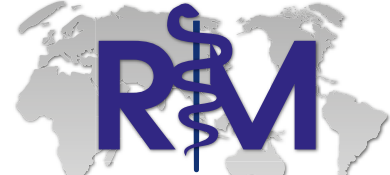 \\ für REISEMEDIZIN e.v. \\ German Society for Travel Medicine}

Hansaallee 299, 40549 Düsseldorf

Tel.: 0211/5202581 Fax: 0211/5202583

E-Mail: info@fachgesellschaft-reisemedizin.de www.fachgesellschaft-reisemedizin.de

\title{
Liebe Kolleginnen und Kollegen, verehrte Mitglieder der DFR,
}

wer das Tagesgeschehen in den Medien aufmerksam verfolgt, stößt immer wieder auf reisemedizinische Bezüge. So jetzt im Zusammenhang mit dem frühen und sehr bedauerlichen Tod von Philipp Mißfelder, dem außenpolitischen Sprecher der CDU-Bundestagsfraktion, an einer Lungenembolie. Dazu haben sich auch außerhalb der Fachöffentlichkeit mehrere Hämatologen gemeldet und auf die hohe Reisetätigkeit und die zahlreichen Interkontinentalflüge des Politikers hingewiesen. Eine eventuell vorliegende familiäre Thromboseneigung sollte entweder an eine Faktor-V-LEIDEN-Mutation (APC-Resistenz) oder an einen ProthrombinDimorphismus denken lassen; beide seien hereditär und innerhalb einer Woche im Blut nachweisbar. Daran müsse, vor allem beim Auftreten von tiefen Beinvenenthrombosen unter 45 Jahren, gedacht werden.

\section{Malariaimpfstoff in Sicht?!}

Die Europäische Arzneimittelagentur EMA hat am 24. Juli ein positives wissenschaftliches Votum für einen Malaria-

\section{Einladung zur Mitgliederversammlung am 18.09.2015}

Hiermit ergeht die Einladung zur diesjährigen ordentlichen Mitgliederversammlung der DFR am Freitag, 18.09.2015, ab 17:15 Uhr, in den Räumen des Swissôtel Dresden.

Tagesordnung:

1. Begrüßung und Feststellung der ordnungsgemäßen Einberufung

2. Ggf. Entscheidung über die Annahme fristgerecht (d. h. bis spätestens eine Woche vor der MV) eingegangener Anträge zur Tagesordnung

3. Feststellung der Tagesordnung

4. Bericht des Präsidenten der DFR o.V.i.A. zu den Aktivitäten der DFR seit der letzten MV

5. Bericht aus dem Vorstand zu Aktivitäten der Fachausschüsse und Arbeitsgruppen

6. Bericht des Kassenwarts und Feststellung Abschluss des Geschäftsjahrs 2014

7. Bericht der Kassenprüfer

8. Entlastung des Vorstands

9. Feststellung des Haushaltplans 2015/2016

10. Diskussion der vorgeschlagenen Beitragsordnung, Beschlussfassung

11. Bericht zu evtl. erforderlichen Satzungsänderungen; Beschlussfassung hierzu

12. Jahrestagungen 2016,2017

13. Verschiedenes (ggf. Bekanntgabe über die Verleihung des Erich-Kröger-und-KlausJörg-Volkmer-Preises)

Zu Punkt 10 der vorgeschlagenen Tagesordnung: Im Zusammenhang mit dem laufenden Antrag zur Anerkennung der Gemeinnützigkeit kann sich die Notwendigkeit von Satzungsänderungen ergeben. Hierüber werden sie noch rechtzeitig mit Rundschreiben informiert, ebenso über den Inhalt der vorgeschlagenen Beitragsordnung.

Bietigheim-Bissingen, am 17.07.2015

Gez. Prof. Dr. Günter Schmolz, Präsident der DFR impfstoff abgegeben, der bisher als RTS,S/AS01 bekannt war und jetzt unter der Bezeichnung Mosquirix hoffentlich den Weg bis zu Zulassung findet. Auch das PEI sieht darin einen wichtigen ersten Schritt. Die WHO will sich bis zum Jahresende äußern, www.ema.europa. eu/docs/en_GB/document_library/ Press_release/2015/07/WC500190447. pdf

Der Impfstoff ist für Kleinkinder in Malaria-Endemiegebieten südlich der Sahara entwickelt und dort an dieser Altersgruppe getestet worden. Wegen seiner eingeschränkten Wirksamkeit, die je nach Beobachtungszeit deutlich unter $50 \%$ liegt, wird er für die Reisemedizin kaum Bedeutung erhalten.

Neuer Service auf unserer Homepage $\mathrm{Ab}$ sofort bietet die DFR auf ihrer Homepage (www.fachgesellschaft-reisemedizin. de) einen neuen Service an. Jeden Monat werden aus der Vielzahl neu erschienener Originalbeiträge und Übersichtsartikel zu reisemedizinischen Themen besonders relevant erscheinende Artikel mit direktem Link zum Beitrag eingestellt, falls möglich, im PDF-Format. Selbstverständlich handelt es sich dabei nur um solche Publikationen, für die eine Erlaubnis des Copyrightinhabers vorliegt, oder die mit open access gekennzeichnet sind. Da es nicht möglich ist, alle relevanten Zeitschriften fortlaufend im Blick zu haben, bitten wir unsere Mitglieder um Hinweise auf wichtige Publikationen, die wir übersehen haben.

\section{NECTM 2016 in London}

Für die 6. North European Conference on Travel Medicine, 1. bis 4. Juni 2016, London/UK, ist seit Kurzem die ,early bird registration‘ eröffnet (http://nectm.com/). Bitte melden Sie sich als Mitglied der DFR an, die bekanntlich Mitveranstalter ist. Sie erhalten dann den reduzierten Teilnehmerbetrag in Höhe von 395 Britischen Pfund. Die Anmeldung wird erleichtert, wenn Sie Ihre DFR-Mitgliedsnummer parat haben. Der Frühbucherrabatt gilt bis 1. März 2016. Die Frist zur Einreichung von Abstracts für Poster/Vorträge endet am 29. Januar 2016. 
DFR-Tagung 2015 in Dresden

Bitte werfen Sie einen Blick auf das aktuelle Programm unserer wissenschaftlichen Jahrestagung am 18. und 19. September in Dresden auf dieser Seite. Anmeldungen sind jederzeit und auch noch kurzfristig möglich. Die Sächsische Landesärztekammer vergibt für unsere Jahrestagung 13 Fortbildungspunkte. Wer außerhalb des Tagungshotels eine preisgünstige Unterkunft sucht, kann sich mit unserer Geschäftsstelle in Verbindung setzen; die DFR hat eine entsprechende Vereinbarung mit einem Hotel getroffen.

Wer zum Tagungspunkt ,Offene Fragerunde' am Samstagnachmittag vorab Fragen einreichen möchte, kann dies jederzeit bei der Geschäftsstelle tun. Fragen, die sich vor Ort ergeben, werden selbstverständlich auch behandelt - solange die Zeit reicht.

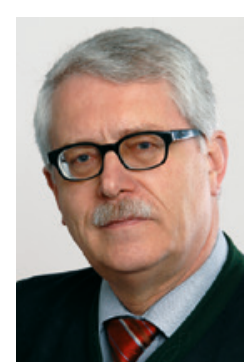

Mit den besten Grüßen Ihr

Günter Schmolz

\section{Jahrestagung der Deutschen Fachgesellschaft für Reisemedizin e. V.}

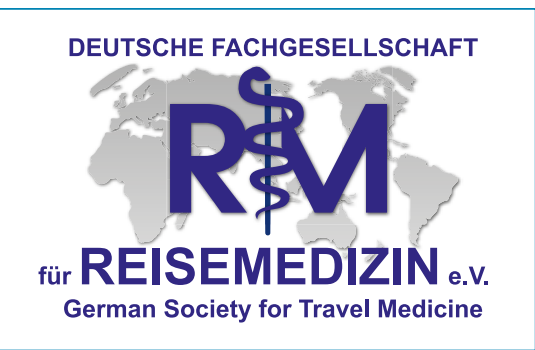

18.-19.09.2015 Dresden

Veranstaltungsort: Swissôtel Dresden Am Schloss · Schloßstraße 16 • 01067 Dresden

Freitag, 18. September

12:30 Uhr

13:00-13:15 Uhr Begrüßung und Eröffnung

der 18. Jahrestagung

Prof. Dr. Günter Schmolz, Bietigheim-Bissingen

13:15-14:00 Uhr Kasuistiken aus der Reisemedizin Dr. Rosemarie Mazzola, Freiburg

14:00-15:00 Uhr Aktuelle Weltseuchenlage neue Impfstoffe Prof. Dr. Tomas Jelinek, Berlin/Düsseldorf

15:00-15:20 Uhr Kaffeepause

15:20-16:45 Uhr Reiseland Indien -

Was muss der Reisemediziner wissen? Impulsvorträge und Expertenpanel zur Beantwortung von Fragen aus dem Auditorium Moderation: Dr. Uschi Traub, Ludwigsburg

16:45-17:15 Uhr

17:15-18:30 Uhr

Reisemedizinische Informationsquellen Andreas Kaunzner, Aschaffenburg

18:30 Uhr

\section{Mitgliederversammlung}

Stadtrundgang mit anschließendem gemeinsamen Abendessen im Restaurant Pulverturm an der Frauenkirche

Die Veranstaltung wurde von der Sächsischen Landesärztekammer mit 13 Fortbildungspunkten zertifiziert.
Samstag, 19. September

09:00-09:30 Uhr Reisen mit kardialen Vorerkrankungen Dr. Ilse Janicke, Duisburg

09:30-10:00 Uhr Reisen mit pulmonalen Vorerkrankungen N.N.

10:00-10:40 Uhr Polymedikation bei älteren Reisenden Prof. Dr. Jürgen Brockmöller, Göttingen

10:40-11:00 Uhr Kaffeepause

11:00-11:45 Uhr Immunsuppression und Impfungen Prof. Dr. Emil C. Reisinger, Rostock

11:45-12:30 Uhr Chronisch kranke Kinder auf Reisen Dr. Martin Alberer, München

12:30-13:30 Uhr Mittagspause

13:30-14:00 Uhr Impfung bei unbekanntem oder unvollständigem Impfstatus - Impfungen in der Schwangerschaft Dr. Jörg Wendisch, Dresden

14:00-15:00 Uhr Ebola - Versuch einer ersten Bilanz aus reisemedizinischer Sicht Dr. Dr. Günter Pfaff, Stuttgart

15:00-15:40 Uhr Offene Fragerunde

Mitglieder des Vorstandes und des wissenschaftlichen Beirates der DFR

15:40-16:00 Uhr Kaffeepause

16:00-16:30 Uhr

Abrechnung reisemedizinischer Leistungen Dr. Burkhard Rieke, Düsseldorf

16:30-17:15 Uhr Reisemedizinisch relevante Rechtsfragen für die ärztliche Praxis aktuelle BGH-Entscheidungen, Gesetzesänderungen Anwaltskanzlei Dr. Weidemann, Dresden

17:15 Uhr
Ausblick und Verabschiedung

Prof. Dr. Günter Schmolz, DFR 